und wobei es sich um nichts anderes als um unseren Unterleibstyphus gehandelt hat. Roederer und Wagler haben ein langes Kapitel ihrer Arbeit den Ursachen der Krankheit gewidmet. In der Stadt Göttingen haben damals allerdings Zustände geherrscht, welche jeder Beschreibung spotten. Unter den in Betracht kommenden Schädlichkeiten spielten der Genuss verdorbenen Fleisches, unreinen Wasser's eine Rolle; freilich ist daneben eine recht grosse Reihe anderer Krankheitsursachen aufgezählt, deren Bedeutung schwieriger erfasst werden kann. Jedenfalls hat es aber angesichts der grossen Nothlage, in der sich die Stadt damals befand, durchaus an Hilfsmitteln gefehlt, den grossen hygieinischen Mängeln wirksam zu begegnen. Die therapeutischen Maassnahmen, welche gegen diesen "morbus mucosus" und die anderen damals in epidemischer Ausbreitung herrschenden Krankheiten gebraucht wurden, erwähnen die Abstellung der vorhandenen Uebelstände als Mittel zur Beseitigung dieser Krankheiten ebensowenig, wie bei der Schilderung der Dysenterie, aus der sich der Morbus mucosus entwickelt haben soll. Uebrigens sind in jener Zeit offenbal auch in anderen Ländern die Ruhr und der Flecktyphus neben dem Unterleibstyphus aufgetreten. Ueber den weiteren Verlauf des Unterleibstyphus in Göttingen im 18. Jahrhundert und in den ersten 64 Jahren des 19. Jahrhunderts liegen mir genauere Nachrichten nicht vor. Es ist in dieser Beziehung nur berichtet, dass seit dem Jahre 1858 die Stadt Göttingen von theils grösseren, theils kleineren Typhusepidemieen heimgesucht worden ist. Diese Erkrankungen setzten meist im Beginn des Monats August ein, und erst im Monat October pflegte die Zahl der Tyhuserkrankungen abzunehmen. Aus dem Jahre 1865 werden aus der damals noch nicht 14000 Einwohner zählenden Stadt Göttingen - die Zahl der Einwohner hat sich seitdem mehr als verdoppelt - 275 Typhuserkrankungen gemeldet. Es erkrankten damals 19\% der Bewohner Göttingens am Unterleibstyphus, ein Verhältniss, das nachher nie wieder erreicht worden ist. Seit dem Jahre 1864 besitzen wir, wenn auch nicht ununterbrochene Angaben über die in den einzelneı Jahren in Göttingen vorgekommenen Typhusfälle. Diese Zahlen entstammen, soviel ich weiss, den Aufzeichnungen der Aerzte. Aus den Jahren $1871-1874$ fehlen bestimmte Zahlenangaben ganz. Es wird nur angegeben, dass im Jahre 1871 die Zahl der Erkrankungen und der Todesfälle am Unterleibstyphus eine grosse gewesen sei. Auch aus dem Jahre 1874 und 1876 wird von Typhusepidemieen in Göttingen berichtet. Die erstere war eine heftige, aber eine lokal beschränkte. Ich habe es mir angelegen sein lassen, seitdem ich im Jahre 1877 die Direction der medicinischen Klinik übernommen habe, die in Göttingen aufgetretenen Typhusepidemieen in Dissertationen beschreiben zul lassen. Ich werde nachler einige Angaben darïber machen und bei dieser Gelegenheit einige Zahlen (cf. auch in Tabelle I die von 1877--1882 aus der Stadt Göttingen gemeldeten Typhusfälle) über die damalige Typhusfrequenz in Göttingen mittlıeilen. Es ist wohl keinem Zweifel unterworfen, dass diese Zahlen viel zll niedrig sind. Die Verordnung vom 20. November 1882 erst legte den Aerzten die Verpflichtung auf, die in ihre Behandlung gelangenden Fälle von Unterleibstyphus der Behörde anzuzeigen. Absolute Zahlen erhält man dadurch freilich auch nicht. Indessen dürfte man auf diese Weise wenigstens einen Ueberblick über die Zahl der schwereren Typhuserkrankungen bekommen. Die Polizeidirektion der Stadt Göttingen hat die Güte gehabt, mir das in den Akten befindliche Material über die Zahl der gemeldeten Typhusfälle an die Hand zu geben (cf. Tabelle I die Typhusfälle aus der Stadt seit 1883 ; aus dem Landkreis seit 1887). Auch die Tabelle II (s. u.) sowie einige andere, die mannigfachen hygieinischen Maassnahmen in der Stadt Göttingen betreffenden Mittheilungen verdanke ich derselben Behörde.

Die Einrichtung der hier beigefügten Tabelle I bedarf nur insofern einer Erläuterung, als in derselben überdies in den letzten drei Rubriken auch eine Uebersicht über die in meiner Klinik seit dem Jahre 1877 behandelten Fälle von Unterleibstyphus gegeben ist, wobei darauf Rücksicht genommen wurde, ob sie aus der Stadt Göttingen selbst oder aus dem Landkıeise Göttingen oder ausserhalb desselben stammen. Ersehen wir nun aus der Tabelle I, einen wie grossen Antheil der Landkreis Göttingen an der Zahl der Typhusfälle in unserem Bezirke hat, so werden wir aus Tabelle II die Ueberzeugung gewinnen, dass auch die ausserhalb des Landkreises Göttingen in einzelnen Dörfern der angrenzenden Kreise vorhandenen Typhuserkrankungen nicht ohne Einfluss auf I unsere Typhusfrequenz sein düriten.

1) J. G. Roederer und C. G. Wagler, De morbo mucoso liber singularis. Goettingae 1762 .

$\left.{ }^{2}\right)$ Jo. H. Riepenhausen, Morbi epidemii etc. ab anno 1757 usque ad 1762 Goettingae et circa eam grassati. Halae 1764. 
Tabelle I.

\begin{tabular}{|c|c|c|c|c|c|c|c|}
\hline & \multirow[b]{2}{*}{$\begin{array}{c}\text { Bei der } \\
\text { Polizei- } \\
\text { direktion } \\
\text { ange- } \\
\text { meldete } \\
\text { Fälle aus } \\
\text { der Stadt } \\
\text { Göttingen } \\
1 .\end{array}$} & \multirow[b]{2}{*}{$\begin{array}{c}\text { Bei dem } \\
\text { Landraths- } \\
\text { ante } \\
\text { ange- } \\
\text { meldete } \\
\text { Fälle aus } \\
\text { dem Land- } \\
\text { kleise } \\
\text { Göttingen } \\
2 .\end{array}$} & \multicolumn{5}{|c|}{$\begin{array}{l}\text { In der medicinichen Klinik behan- } \\
\text { delte Fälle }\end{array}$} \\
\hline & & & \begin{tabular}{|c|} 
\\
aus \\
der \\
Stadt \\
Göt- \\
tingen \\
3.
\end{tabular} & $\begin{array}{c}\text { aus } \\
\text { dem } \\
\text { Land- } \\
\text { kreise } \\
\text { Göt- } \\
\text { tingen } \\
4 .\end{array}$ & $\begin{array}{c}\text { aus be- } \\
\text { nachbarten } \\
\text { Kreisen: } \\
\text { Münden, } \\
\text { Northeim, } \\
\text { Duderstadt, } \\
\text { Heiligen- } \\
\text { stadt } \\
5 .\end{array}$ & $\begin{array}{c}\text { aus } \\
\text { wei- } \\
\text { terer } \\
\text { Fnt- } \\
\text { fer- } \\
\text { nung } \\
\text { zuge- } \\
\text { reiste } \\
6 .\end{array}$ & $\mid \begin{array}{ll}0 \\
0 \\
1 & 0 \\
0 & 0 \\
0 & 1 \\
0 & 0 \\
0 & 0 \\
3 & a \\
3 & 0 \\
0 & 0\end{array}$ \\
\hline 1877 & 85 & - & 20 & 6 & 2 & 2 & 30 \\
\hline 1878 & 135 & - & 31 & 6 & 3 & 1 & 41 \\
\hline 1879 & 31 & $\ldots$ & 10 & 9 & 2 & 2 & 29 \\
\hline 1880 & 57 & - & 10 & 7 & 4 & 3 & 24 \\
\hline 1881 & 106 & - & 23 & 4 & 6 & 5 & 38 \\
\hline 1882 & 32 & - & 11 & 7 & 5 & 3 & 26 \\
\hline 1883 & $\left.64^{\prime}\right)$ & - & 18 & 9 & 9 & 1 & 37 \\
\hline 1884 & $25^{\prime}$ & - & 10 & 11 & 8 & 10 & 39 \\
\hline 1885 & 48 & - & 10 & 6 & 6 & 3 & 25 \\
\hline 1886 & 58 & - & 32 & 7 & 5 & 4 & 48 \\
\hline 1887 & 26 & 29 & 15 & 10 & 2 & 5 & 32 \\
\hline 1888 & 29 & 19 & 13 & 9 & 5 & - & 27 \\
\hline 1889 & 60 & 60 & 19 & 7 & 4 & 2. & 32 \\
\hline 1890 & 9 & 32 & 6 & 3 & 3 & 1 & 13 \\
\hline 1891 & 14 & 24 & 3 & 1 & 4 & 2 & 10 \\
\hline 1892 & 5 & 11 & 2 & 2 & 1 & - & 5 \\
\hline 1893 & 25 & 20 & 6 & 3 & 1 & - & 10 \\
\hline 1894 & 19 & 101 & 8 & 11 & - & 1 & 20 \\
\hline 1895 & 6 & 28 & 7 & 3 & 6 & 1 & 17 \\
\hline 1896 & $?$ & 10 & 1 & 5 & 1 & 1 & 8 \\
\hline 1897 & 14 & 15 & 7 & 6 & 2 & 4 & 19 \\
\hline 1898 & 7 & 13 & 6 & 3 & 2 & 1 & 12 \\
\hline 1899 & 19 & 12 & 4 & 3 & 1 & 1 & 9 \\
\hline 1900 & 52 & 20 & 49 & 7 & 6 & 一 & 62 \\
\hline $\begin{array}{l}\text { bis } 15 \text {. No } \\
\text { vember }\end{array}$ & & & & & & & \\
\hline
\end{tabular}

Ich bespreche jetzt die während meiner Amtsführung als Direktor der medicinischen Klinik in Göttingen aufgetretenen Typhusepidemieen und -Endemieen.

Nach dem Erlöschen der bereits erwähnten epidemischen Ausbreitung des Typhus im Jahre 1876 mit 142 Fällen sank im Jahre 1877 die Zahl der Typhuserkrankungen auf 85 Fälle, wovon fünf starben. Nur der Monat November war frei von Erkrankungen. Die meisten Typhusfälle entfielen, zu einer etwas grösseren Hausepidemie anschwellend, auf den Herbst des genannten Jahres. Im September und October des Jahres 1877 nämlich erkrankten in dem auf der unteren Masch gelegenen Waisenhause, in welchem damals 35 Kinder (20 Knaben und 15 Mädchen) verpflegt wurden, zehn dieser Waisenkinder (sechs Knaben und vier Mädchen) und ausserdem die Tochter des Inspektors des Waisenhauses am Unterleibstyphus. Sämmtliche zehn Waisenkinder wurden in der medicinischen Klinik behandelt. Ein zehnjähriges Mädchen, bei welchem der Typhus mit Lungenentzündung und hämorrhagischer Diathese complicirt war; erlag der Krankheit. Auch bei dieser kleinen Typhusendemie kam man, wie bei so vielen anderen, betreffs ihrer Entstehung über Vermuthungen nicht hinaus. Am wahrscheinlichsten erscheint der Zusammenhang dieser Endemie mit der sogenannten Kuhleine - d. i. einem Arm des durch die Stadt Göttingen fliessenden Leinekanals -, welche, die Abfälle der anliegenden Grundstücke aufnehmend, wieder in den Leinekanal einfliesst. Auch alle Abwässer des damals an der Kuhleine gelegenen pathologischen Instituts flossen in diesen Wasserarm. Das Trinkwasser, welches einem Brunnen auf dem Hofe des Waisenhauses entnommen wurde, ergab absolut keine Anhaltspunkte für die Annahme, dass darin die Ursache der Typhusendemie zu suchen sei. Jedenfalls konnte aber betreffs des Antheils, den die Kuhleine an der Entstehung der Typhusendemie hatte, so viel festgestellt werden, dass die in die Kuhleine führende, im Hofe des Waisenhauses gelegene Abzugsgosse offen und zu Zeiten mit faulenden, widerwärtig riechenden Massen so sehr angefüllt war, dass nicht nur im Hofe, sondern auch im Hause selbst ein höchst unangenehmer Gestank entstand. Der sichere Nachweis aber, auf welchem Wege und auf welche Weise es zur Entwickelung des Unterleibstyphus gekommen ist, konnte nicht erbracht werden. Bemerkenswerth ist immerhin, dass vorher in den der Kuhleine anliegenden Häusern, in der sogenannten Neustadt, die oberhalb der Kuhleine liegt, Typhusfälle vorgekommen waren. Unschwer ist jedenfalls die

1) 8 davon im Göttinger Garnisonlazareth behandelt.
Verbreitung des Typhus bei dem engen Zusammenleben so vieler Kinder zu verstehen.

Das Jahr 1878 brachte wieder mehr Typhuserkrankungen als das vorhergehende Jahr, nämlich 132 Fälle, wovoll acht starben. Die Erkrankungen vertheilten sich ungleich über die einzelnen Monate. Auf die Monate August bis Oktober entfielen wohl die meisten Fälle, nämlich 52. Es wurde behauptet, dass zll dieser Zeit Unterleibstyphus aus Hamburg eingeschleppt worden sei Jedenfalls waren aber auch ohnedies nach wie vor in Göttingen alle Umstände vereinigt, welche der autochthonen Entstehung des Typhus Vorschub leisten konnten. Bemerkenswerth erscheint im Jahre 1879 die im Vergleich mit dem Vorjahre geringe Zahl der Typhusfälle: 31 mit vier Todesfällen. Die Fälle waren über die ganze Stadt zerstreut. Die geringe Zahl der Typhuserkrankungen war schwer zu deuten. Das Trinkwasser war noch nicht besser geworden, in die Gossen der Strassen wurden noch Fäkalien u. s. w. nach wie vor ausgeschüttet, der Grundwasserstand war denselben Schwankungen unterworfen wie früher. Als bemerkenswerther Unterschied mag hervorgehoben werden, dass der Erdboden in diesem Jahre nie so trocken war wie in den vorhergehenden Jahren, weil die atmosphärischen Niederschläge erheblich reichlicher waren als in diesen. Auch waren die Tagesschwankungen der Temperatur geringer als in den Vorjahren. Eine befriedigende Erklärung für die geringe Typhusmorbidität in diesem Jahre ist damit freilich nicht gegeben.

Im Jahre 1880, mit 57 Typhuserkrankungen und vier Todesfällen, traten die meisten Erkrankungen im März und April, nämlich 28, auf, und zwar wegen einer kleinen Epidemie am Bahnhof, welche mit dem Wasser eines Pumpbrunnens in Verbindung gebracht wurde, in welchem sich jedoch bei der Untersuchung nichts Verdächtiges vorfand. Indess, nachdem der Brunnen nichtsdestoweniger geschlossen wurde, erlosch die Epidemie. Die übrigen Fälle waren über die Stadt zerstreut.

Das folgende Jahr 1881 brachte wieder eine viel grössere Zahl von Typhuserkrankungen, welche aber fast durchweg einen günstigen Verlauf nahmen. Im Jahre 1881 nämlich traten in der Stadt Göttingen 106 Typhuserkrankungen auf, von denen nur drei tödtlich verliefen. Von den 106 Typhuskranken entfielen 72 auf die Monate Juli und August des genannten Jahres. Von diesen 72 Typhuskranken starb indes nur einer. Der Rest von 32 Fällen vertheilte sich ziemlich gleichmässig über die übrigen zehn Monate des Jahres 1881. Bei dieser Epidemie war bemerkenswerth, dass in manchen Häusern mehrere, oft verhältnissmässig viele Personen am Typhus erkrankten, und diese Thatsache wurde um so bemerkenswerther dadurch, dass die Bewohner dieser Häuser aus solchen Brunnen das Wasser zum Trinken und zum Bereiten der Speisen benutzt hatten, welche erwiesenermaassen organische oder sonstige für den menschlichen Körper schädliche Bestandtheile enthielten. Bei 51 Kranken konnte kein anderer Grund für die Krankheit gefunden werden als gerade die Pumpbrunnen, die schon in früheren Jahren als krankheits- und speziell als typhuserregend bezeichnet werden mussten. In einem Hause (Weenderstrasse 10) erkrankten sechs Personen am Typhus. Das Trinkwasser des diesem Hause gegenüberliegenden Brunnens, aus dem die Bewohner regelmässig das ihrige bezogen, musste als die Ursache für diese Erkrankungen angesehen werden. Dem Grundwasserstande konnte bei dieser Epidemie keine Schuld an den Typhuserkrankungen gegeben werden. Derselbe wurde dreimal monatlich an neun Pumpbrunnen gemessen. Dabei hat sich ergeben, dass derselbe das ganze Jahr hindurch nur geringe Schwankungen erfahren hat. Die folgenden Jahre brachten einen sehr wesentlichen Rückgang der Typhuserkrankungen, welche von vielen Seiten schon mit der allerdings in rüstigem Fortgange begriffenen Besserung der hygieinischen Verhältnisse in Göttingen, wovon später die Rede sein wird, in Zusammenhang gebracht wurden. Die Steigerung der Zahl der Typhuserkrankungen im Jahre 1886 auf 86 rührt wenigstens zum Theil von einer interessanten kleinen Hausendemie her. Im November und Dezember 1886 traten unter den 50 Bewohnern der vier Häuser Groner Chaussee 16 B bis E, welche einen Gebäudecomplex bilden, 17 Erkrankungen an Unterleibstyphus auf. Es stellte sich heraus, dass der gemeinsame Brunnen dieser vier Häuser, dessen Wasser. zum Trinken, zum Kochen und zu allen Zwecken des häuslichen Gebrauches diente, gegen den mit einem ausserordentlich stinkenden Inhalt angefüllten Schlammkasten nicht vollständig abgedichtet war, sodass von ihm Abwässer in den Brunnenraum hineinliefen. Das Wasser diẹses Brunnens ergab sich bei der Unter- 
suchung als ein sehr verunreinigtes, was freilich unter diesen Umständen nicht anders erwartet werden konnte. Jedoch liess sich ein direkter Beweis dafür, dass das Wasser bei der typhösen Infection den wirklichen Krankheitserreger enthalten habe, nicht erbringen. Von diesen 17 Typhusfällen sind je sechs männliche und weibliche Patienten in der medicinischen Klinik behandelt worden. Von ihnen starb eine 38jährige Fraul, aber nicht sowohl direkt an dem Typhus oder an der gleichzeitig daneben bestehenden Phthise, sondern insbesondere in Folge der schweren Blltungen aus den Geschlechtsorganen, welche bei der im zweiten Monat der Schwangerschaft befindlichen Patientin auftraten. Bemerkenswerth war bei dieser Endemie die Neigung zu Recidiven, welche bei nicht weniger als fünf der 12 klinischen Patienten beobachtet wurden. In einem der Fälle, welcher ein achtjähriges Mädchen betraf, wurde sogar ein zweimaliges Recidiv beobachtet.

Erst seit dem Jahre 1890 hat die Zahl der Typhuserkrankungen in Göttingen dauernd abgenommen, insbesondere ist eine Epidemie seitdem bis zum Somnier 1900 nicht mehr aufgetreten. Diese Abnahme der Typhuserkrankungen fällt mit der Einrichtung einer Reihe hygieinischer Maassnahmen zusammen, welche in Göttingen in Angriff genommen, bezw. ausgefïhrt worden waren. Davon ist in erster Reihe die Versorgung der Stadt mit gutem Trinkwasser $\mathrm{zu}$ erwähnen. Das Trinkwasser der Göttinger entstammte bis zum Ende der siebziger Jahre des verflossenen Jahrhunderts fast ausschliesslich aus den Pumpbrunnen, welche auf den Höfen der Häuser und in den Strassen der Stadt aufgestellt waren. Die Untersuchuug des Wassers, welches diese Brunnen lieferten, hat ergeben, dass es meist als Trinkwasser unbrauchbar war. Die Versorgung der Stadt Göttingen mit gutem Trinkwasser vollzog sich allmählich. Das Hochdruckbassin der Wasserleitung ist im Jahre 1877 gebaut. Die Verstärkung der Wasserleitung durch das Pumpwerk bei der Stegemühle ist im Jahre 1892 erfolgt. Auf diese Weise wurde den Bewohnern der Stadt Göttingen allerdings die Gelegenheit gegeben, sich mit gutem Trinkwasser zu versorgen, und es wurde auch bald ein ziemlich ausgiebiger und stetig zunehmender Gebrauch davon gemacht. Indes wurde auch von einer Reihe der den besseren Ständen angehörigen Personen Pumpbrunnenwasser zum Trinken benutzt. Zwar waren die auf den Strassen der Stadt befindlichen Pumpbrunnen allmählich beseitigt worden, doch bestand noch eine grosse Reihe von Pumpbrunnen in den Höfen der Häuser sogar bis in die allerjüngste Zeit, und einzelne Menschen, denen man eine bessere Einsicht zutrauen sollte, haben dasselbe bis jetzt ausschliesslich getrunken. Dass jetzt - - während der Typhusepidemie im Sommer 1900 war der Schluss aller Pumpbrunnen verfïgt worden - wirklich alle Pumpbrunnen in der Stadt Göttingen beseitigt sind, scheint mir nicht genügend sichergestellt. Geschehen aber muss es unter allen Umständen. Jeder derartige in Funktion befindliche Brunnen ist direkt gemeingefährlich. Seine Verunreinigung mit Krankheitskeimen, insbesondere auch mit Typhusgift, bildet zwar nicht die alleinige, aber doch eine sehr häufige Ursache für die Enstehung von Seuchen, besonders auch für die des Unterleibstyphus.

\section{(Schluss folgt.)}

Blaschko, Hygiene der Prostitution und venerischen Krankheiten. 40. Lieferung des Handbuchs der Hygiene, herausgegeben von Theodor Weyl. Jena, Gustav Fischer, 1900. 128 S., 3,00 M. Ref. Klingmüller (Breslau).

An der Hand eigener reicher Erfahrungen und zahlreicher statistischer Erhebungen aus den kultivirten Ländern giebt uns der bekannte Verfasser im vorliegenden Buche eine ausgezeichnete Uebersicht über diesen Theil der Hygiene. Das Buch ist klar geschrieben; das Für und Wider der Ansichten wird einer objektiven Beurtheilung unterzogen; eine Menge Vorurtheile, die auch hente noch in der "Gesellschaft" und Wissenschaft als endgiltige Wahrheiten vorgetragen werden, werden mit schonungsloser Kritik und wissenschaftlichem Ernst zerstört. Darum sei das Buch allen denen, die menschliches und wissenschaftliches Interesse diesem trostlosen Kapitel der 'sozialen Hygiene entgegenbringen, warm empfohlen.

Der Verfasser bespricht zunächst die Prognose und Verbreitungsweise der Syphilis und Gonorrhoe, welche hauptsächlich als Volkskrankheiten in Betracht kommen, im Anschluss daran die Verbreitung der Geschlechtskrankheiten. Der hohe Prozentsatz sei an folgenden Zahlen illustrirt:

Deutsche Armee 1877-1896: 35,3-25,5\%

Deutsche Marine 1877-1885: 139,0\%

Desgl. (ostasiat. Bes.) 1881-1882: 289,7\%
Kaufleute (berechnet nach einer ïber ganz Deutschland verbreiteten Krankenkasse) 1893-1897: 8,0-8,2\%

In den Grossstädten steigt diese Zahl bis auf dreifache (Breslau: $27,8 \%$ ). Davon entfallen auf Tripper $70-75 \%$, auf Ulcus molle und Syphilis $25-30 \%$.

Im dritten Theil behandelt der Verfasser die Prostitution. Seine Ansichten lassen sich kurz durch folgende Sätze wiedergeben: „Stehen wir eben auf dem Standpunkt, dass nicht das Angebot von Prostitution ein Bedürfniss erzeugt, sondern dass das nach Befriedignng lechzende gesellschaftliche Bedürfniss erst das Angebot provozirt, so müssen die ausschliesslich auf Verringerung des Angebotes, bezw. des Zustroms zur Prostitution gerichteten Bestrebungen erfolglos bleiben. Sie können höchstens den Erfolg haben, dass die anderen Formen des ausserehelichen Geschlechtsverkehrs in den Vorderorund treten."

Den Hanpttheil des Buches bildet die Prophylaxe der vene. rischen Krankheiten. Nach einer geschichtlichen Einleitung bespricht der Verfasser die Reglementirung, wie sie in den verschiedenen Ländern und Städten gehandhabt wird, die polizeiliche nnd sanitäre Beaufsichtigung der Bordelle und der einzeln lebenden Prostituirten. Die Resultate der Reglementirung hält der Verfasser für schlecht Jedenfalls lässt sich nach dem vorliegenden statistischen Material nicht der Schluss ziehen, dass die Untersuchung und Behandlung der polizeilich eingeschriebenen und uberwachten Prostituirten einen günstigen Einfluss auf die Gesundheit der männlichen Bevölkerung ansübt. Die Mängel der Reglementirung liegen darin, dass von den wirklich gewerbsmässigen Prostituirten zu wenig eingeschrieben sind, dass die Untersuchung wegen Mangel an geschulten Untersuchern und wegen Mangel an Zeit nicht eingehend genug ist, dass die Behandlung nicht gründlich ist und dass die Reglementirung gewisse ungünstige Nebenwirkungen hat (Härte der Gerichtsstrafen, Bekanntwerden mit de Sittenpolizei u. a.).

Eine vollkommene Assanirung der Prostitution ist heute und au absehbare Zeit völlig unmöglich.“ Zur Besserung des jetzt bestehenden Uebelstandes hält der Verfasser folgendes für nothwendig:

Die Behandlung von Geschlechtskrankheiten muss möglichst unentoeltlich geschehen. Die Polizei darf Mädchen allein deswegen, weil sie eine Geschlechtskrankheit erworben haben oder deswegen behandelt werden, nicht weiter beaufsichtigen. Dagegen müssten auf Grund einer Gesetzesbestimmung zwangsweise untersucht werden können Personen beiderlei Geschlechtes, welche 1. denınzirt wurden, weil sie angeblich eine Ansteckung veranlasst haben, 2. wegen eines Vergehens gegen die Sittlichkeit unter Anklage stehen, insbesondere wenn sie „durch eine unzüchtige Handlung ein öffentliches Aergerniss gegeben haben“. Die Behandlung solle dann zwangsweise geschehen und möglichst gründlich und unentgeltlich in geeigneten Krankenhäusern und Polikliniken. Der ärztlichen Untersuchung und Behandlung soll der polizeiliche Charakter genommen werden.

Gleichzeitig müsste aber auch die Prophylaxe der Geschlechtskrankheiten in anderen Bevölkerungsklassen gefördert werden. Als geeignete Mittel erscheinen dem Verfasser folgende: Alle Gesetze und Einrichtungen sind so zu treffen, dass von den Venerischen möglichst viele das Krankenhaus aufsuchen. Dazu ist nothwendig, dass die Krankenkassen an Geschlechtsklankheiten Leidende anderen Patienten gleich stellen und ihnen Behandlung und Aufnahme in ein Krankenhaus, sowie Krankengeld nicht verweigern; dass mehr Krankenhäuser als jetzt Geschlechtskranke aufnehmen. Für den Krankenkassen nicht angehörige Patienten wäre es erwïnschter, eine unentgeltliche Behandlung zu cewähren oder grössere Gemeinden, bezw. den Staat für die Kosten aufkommen zu lassen. Neben diesen wichtigsten Vorschlägen befürwortet der Verfasser noch eine Reihe anderer Maassnahmen, die man an Ort und Stelle nachlesen muss.

Grossen Werth legt der Verfasser ferner darauf, das Volk über alle die ungünstigen Verhältnisse, welche sich aus der Prostitution ergeben, aufzuklären. Im Anschluss daran giebt er Verhaltungsmaassregeln für Syphilitiker und Tripperkranke. Als Anhang sind beigegeben das dänische Gesetz über die Maassnahmen gegen die Verbreitung der venerischen Krankheiten und der norwegische Gesetz. entwurf über die Maassnahmen zur Bekämpfung der venerischen Ansteckung und der öffentlichen Unsittlichkeit.

Die Litteratur ist bei den zugehörigen Abschnitten in aus "ührlichen Zusammenfassungen angeführt 